$$
\text { CONF- } 9608126-2
$$

Invited Paper

NATO Advanced Study Institute on Surface Diffusion: Atomistic and Collective Processes, Rhodes, Greece, August 26 - September 6, 1996

\title{
DIFFUSION AND DISSOCIATION OF TWO-
DIMENSIONAL ISLANDS ON FCC METAL (100) \\ DIFFUSION AND DISSOCIATION OF TWO-
DIMENSIONAL ISLANDS ON FCC METAL (100) \\ SURFACES
}

Zhenyu Zhang, Zhu-Pei Shi, and Kenneth Haug

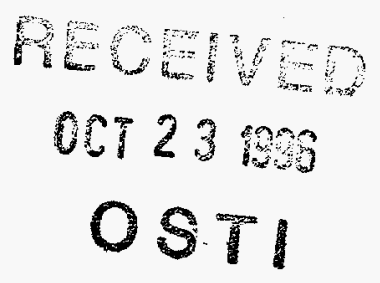

"The submitted manuscript has been authored

by a contractor of the U.S. Government under

contract No. DE-AC05-960R24464.

Accordingly, the U.S. Government retains a

nonexclusive, royalty-free license to publish or

reproduce the published form of this

Government purposes."

OSTI

SOLID STATE DIVISION

OAK RIDGE NATIONAL LABORATORY

Managed by

LOCKHEED MARTIN ENERGY RESEARCH CORP.

Under

Contract No. DE-AC05-960R22464

With the

U. S. DEPARTMENT OF ENERGY

OAK RIDGE, TENNESSEE

September 1996 


\section{DISCLAIMER}

This report was prepared as an account of work sponsored by an agency of the United States Government. Neither the United States Government nor any agency thereof, nor any of their employees, makes any warranty, express or implied, or assumes any legal liability or responsibility for the accuracy, completeness, or usefulness of any information, apparatus, product, or process disclosed, or represents that its use would not infringe privately owned rights. Reference herein to any specific commercial product, process, or service by trade name, trademark, manufacturer, or otherwise does not necessarily constitute or imply its endorsement, recommendation, or favoring by the United States Government or any agency thereof. The views and opinions of authors expressed herein do not necessarily state or reflect those of the United States Government or any agency thereof. 


\section{DISCLAIMER}

Portions of this document may be illegible in electronic image products. Images are produced from the best available original document. 
Invited paper presented at the

NATO Advanced Study Institute on

Surface Diffusion: Atomistic and Collective Processes

Rhodes, Greece, August 26 - September 6, 1996

\title{
DIFFUSION AND DISSOCIATION OF TWO-DIMENSIONAL ISLANDS ON FCC METAL (100) SURFACES
}

\author{
Zhenyu Zhang1, Zhu-Pei Shi1, and Kenneth Haug2 \\ 1Solid State Division \\ Oak Ridge National Laboratory \\ Oak Ridge, TN 37831-6032, USA \\ 2Department of Chemistry \\ Lehigh University \\ Bethlehem, PA 18015, USA
}

\begin{abstract}
The mobility and the stability of small two-dimensional islands on a substrate are basic issues of surface science and thin-film growth. In this article, we present our main results from a series of theoretical studies of island diffusion and dissociation on several fcc metal (100) surfaces, with and without the effects of hydrogen as surface impurities. We found that a collective atomic process, shearing of a dimer belonging to a compact island, in many cases provides the most effective pathway for island diffusion. Consideration of this novel atomic process leads to the possibility of observing a new set of critical island sizes in dynamical island growth or coarsening. When $\mathrm{H}$ is introduced into the Ni system, the mobility of adatoms and islands of all sizes are enhanced. This conclusion suggests that $\mathrm{H}$ will function as an anti-surfactant in $\mathrm{Ni}(100)$ homoepitaxy.
\end{abstract}

\section{INTRODUCTION}

A detailed understanding of the migration of adatoms and islands at surfaces is a prerequisite for development of realistic mechanisms in thin-film growth. Using field ion microscopy (FIM), several groups have investigated the migration of small islands (ranging from 2 to a few tens of atoms in size) at metal surfaces. ${ }^{1-5}$ More recently, the migration of relatively large adatom or vacancy islands (up to a few hundreds of atoms) have been investigated extensively as well, using scanning tunneling microscopy (STM). ${ }^{6-10}$ Additionally, there has been a great deal of interest in exploring the effects of surface impurities in influencing island mobility $6,7,11,12$ and thin-film growth. ${ }^{13-20}$ In particular, it has been shown that some impurities can function as surfactants by inducing smoother film growth, ${ }^{13-19}$ while others as anti-surfactants by deteriorating film qualities. ${ }^{12,13,20}$ 
On the theoretical side, diffusion of islands have been studied by various approaches as well. These include total-energy calculations using first-principles ${ }^{21,22}$ or semi-empirical ${ }^{23-}$ 26 interaction potentials, kinetic Monte Carlo 23,27 and direct molecular dynamics simulations, ${ }^{28}$ and, in the case of large islands, continuum equation methods. ${ }^{29,30}$ These studies have focused on identifying the microscopic diffusion mechanisms involved, and on establishing characteristic relationships such as the dependence of island mobility on island size. For large islands, several competing diffusion mechanisms have been proposed, including periphery diffusion (PD), ${ }^{23}$ evaporation-condensation (EC), ${ }^{23,8}$ and the dislocation mechanism. ${ }^{28}$ In the PD model, the center of mass of an island moves as a result of adatom diffusion along the island periphery. In the EC model, island motion is induced by adatom exchange between islands. In the dislocation mechanism defined on a $\mathrm{fcc}(111)$ surface, island motion is initiated by part of the island collectively shifting from fcc to hep sites. Several recent model studies have also considered the effects of impurities, in particular hydrogen, 31,32 on island mobility and on dynamical island growth.

This article is limited to island diffusion and dissociation on fcc metal (100) surfaces. The purpose is summarize our main results from recent theoretical studies 26,31 of island diffusion and dynamical island growth in these systems, as obtained using the embedded atom methods (EAM). ${ }^{33,34}$ We first consider the diffusion of small islands on a clean surface, where our focus is to present a collective atomic process, dimer shearing, as a novel mechanism for island diffusion. Also discussed are several important implications of this process in dynamical island growth. Next we consider the effects of hydrogen in influencing island stability and mobility, taking the case of $\mathrm{Ni}$ islands on $\mathrm{Ni}(100)$ as an example. It will be shown that the addition of $\mathrm{H}$ onto the surface will reduce the kinetic stability of $\mathrm{Ni}$ islands of all sizes, thereby enhancing the speed of island coarsening. The net effect of $H$ is to function as an anti-surfactant in $\mathrm{Ni}(100)$ homoepitaxy.

\section{ISLAND DIFFUSION ON FCC(100) SURFACES}

When compared with single atom diffusion, island diffusion can proceed via many more different mechanisms. In general, small islands diffuse faster than larger islands, but even very large islands (containing up to hundreds of atoms) can have significant mobility at room temperature. ${ }^{6-12}$ For sizes less than 10 atoms, an oscillatory behavior of the island mobility with island size has been observed on metal (100) surfaces. 5
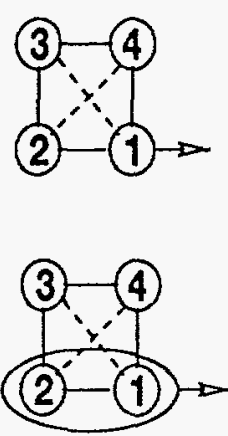

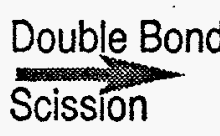

(a)

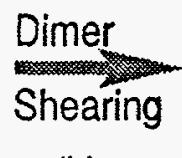

(b)
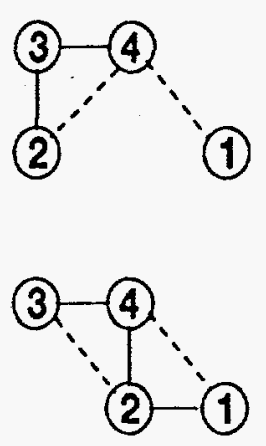

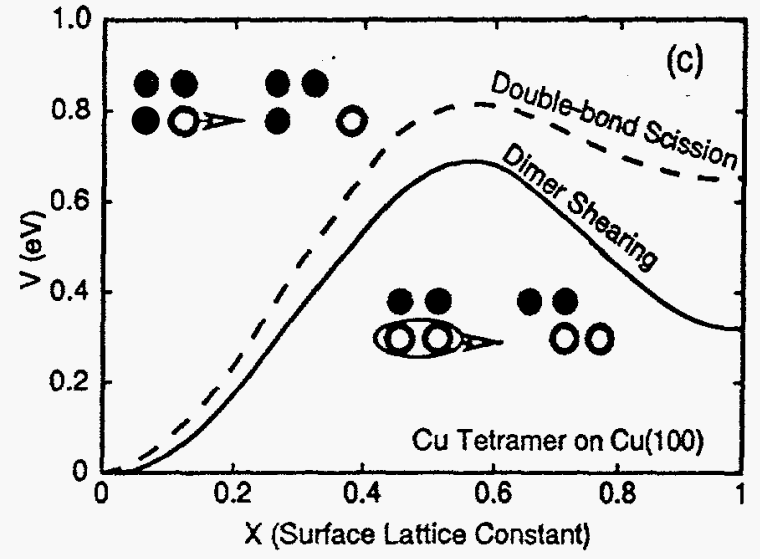

Fig. 1. A comparison of two competing mechanisms leading to the diffusion or dissociation of a tetramer on a $\mathrm{fcc}(100)$ surface: (a) by double-bond scission; (b) by dimer shearing. In (c), the corresponding potential energy surfaces along the two different diffusion pathways are plotted for the case of $\mathrm{Cu}$.

Recently, we have introduced a collective atomic process, shear motion of a dimer belonging to a compact island, as a novel mechanism to initiate diffusion and dissociation of small islands on $\mathrm{fcc}(100)$ surfaces. ${ }^{26}$ A qualitative picture of dimer shearing as a favorable process is described as follows. In the traditional mechanism of sequential motion of individual atoms, diffusion of a tetramer shown in Fig. 1(a) must be initiated by first placing 
atom 1 one step ahead, involving the breakage of two nearest-neighbor (nn) bonds. We denote the corresponding activation energy as $E_{2 b}$. However, the process shown in Fig. $1(b)$, in which one half of the island moves forward together, is expected to be energetically more favorable (i.e., $E_{s}<E_{2 b}$ ), because it involves the breakage of only one nn bond.

In order to confirm this expectation quantitatively, first-principle calculations are apparently preferred to obtain accurate energy barriers, but such calculations are strongly limited by computing power. As an alternative, we have employed the embedded-atom method, ${ }^{33}$ which provides a simple yet reasonable potential that allows exploration of various complicated island diffusion mechanisms at fcc metal surfaces. In these calculations, the substrate is modeled by a slab of 12 layers with 128 atoms per layer. Atoms in the bottom 3 layers are fixed at their respective bulk positions, while atoms in the top 9 layers plus those in the ad-island are free to move. Periodic boundary conditions are applied in the plane parallel to the surface. The potential energy surface along a given reaction path is mapped out by the standard approach: increase the coordinate of the diffusing atom along the reaction path, then at each fixed increment of this coordinate fully relax all the other coordinates of the active atoms. When a diffusion process involves dimer shearing, the coordinates of both atoms of the dimer are increased along the direction of motion, but only one of them is fixed in the relaxation step, allowing a free adjustment of the dimer bond length.

Our detailed EAM calculations confirm the above expectation. Taking the case of a $\mathrm{Cu}$ tetramer on $\mathrm{Cu}(100)$ as an example, the calculated activation energies, $E_{2 b}$ and $E_{s}$, as functions of the displacement indicate that the dimer shear motion is lower in activation energy by $0.13 \mathrm{eV}$ than that of the double-bond scission, as plotted in Fig. 1(c). In fact, Table I shows that in every case where the island size $\mathrm{N}=4,6$, or 8 , and for every element considered ( $\mathrm{Cu}, \mathrm{Ag}$, or $\mathrm{Ni})$, the EAM calculations always support the inequality $E_{s}<E_{2 b}$. One should notice that, the dimer shearing process not only provides a diffusion pathway lower in activation energy, this pathway also contains an "entropic" gain as shown in Fig. 2. In Fig. 2(a), the diffusion pathway of a Ni tetramer on $\mathrm{Ni}(100)$ is initiated by the dimer shear motion, and followed by the shear motion of the other dimer. In this mechanism, it takes only two steps to displace the whole tetramer one surface lattice spacing to the right. The highest activation barrier in this diffusion pathway is only $0.84 \mathrm{eV}$. In contrast, as shown in Fig. 2(b), the traditional picture based on sequential motion of single atoms would require four steps for the same displacement of the tetramer. This mechanism also requires a higher activation energy of $0.99 \mathrm{eV}$. Clearly, the process shown in Fig. 2(a) will dominate the diffusion of the tetramer. This type of diffusion process is also favored for compact islands with sizes of 6 and 8.

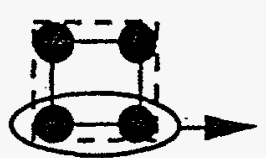

0.84 .

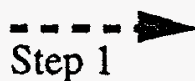

Step 1

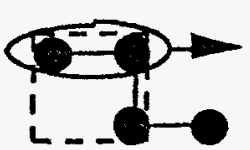

(a)
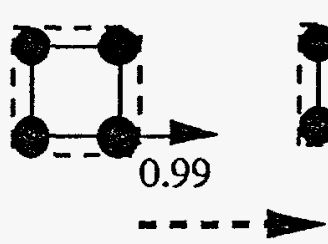

Step 1

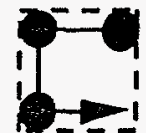

Step 2
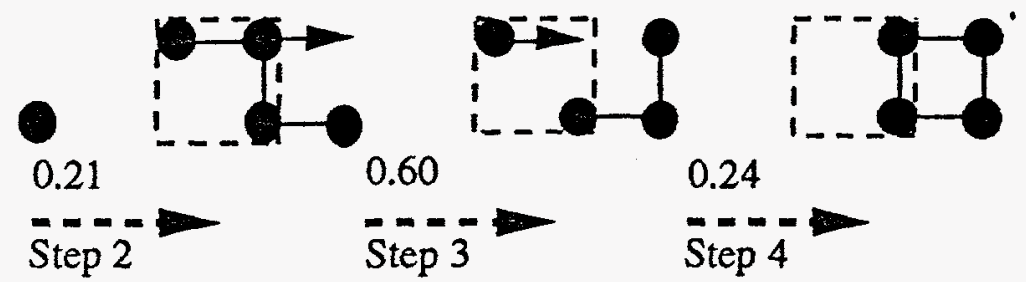

(b)

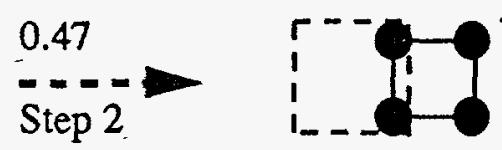

Fig. 2. Diffusion pathways for a $\mathrm{Ni}$ tetramer on a Ni(100) surface via: (a) the new mechanism of dimer shearing; (b) the traditional mechanism of sequential motion of individual atoms. The dashed square marks the original place of the tetramer. The numbers are activation energies in units of $\mathrm{eV}$.

Table I further shows that, the diffusion of islands with a single atom at the island edge (e.g., islands with sizes 5,7 , and 10) is initiated by placing the lone edge atom one step ahead. The corresponding activation energy, $E_{b}$, is lower than $E_{s}$. The calculated activation barriers listed in Table I for adatom and islands consisting of two to ten atoms of $\mathrm{Cu}, \mathrm{Ag}$ and 
$\mathrm{Ni}$ on their (100) surfaces exhibit an interesting oscillatory behavior as a function of island size. Islands with compact geometry (squares and rectangles) have consistently higher activation energies than islands with an extra atom at the periphery. The oscillation magnitude for island sizes between 3 and 8 is on the order of $\left(E_{s}-E_{b}\right)$, or about $0.15-0.20 \mathrm{eV}$ in Fig. 3. This oscillation magnitude is considerably lower than the value of $\left(E_{2 b}-E_{b}\right)$, or about $0.37 \mathrm{eV}$. Notice that the experimentally observed oscillation magnitude of about 0.1 $\mathrm{eV}$ is certainly much closer to $\left(E_{s}-E_{b}\right)$ than to $\left(E_{2 b}-E_{b}\right)$.

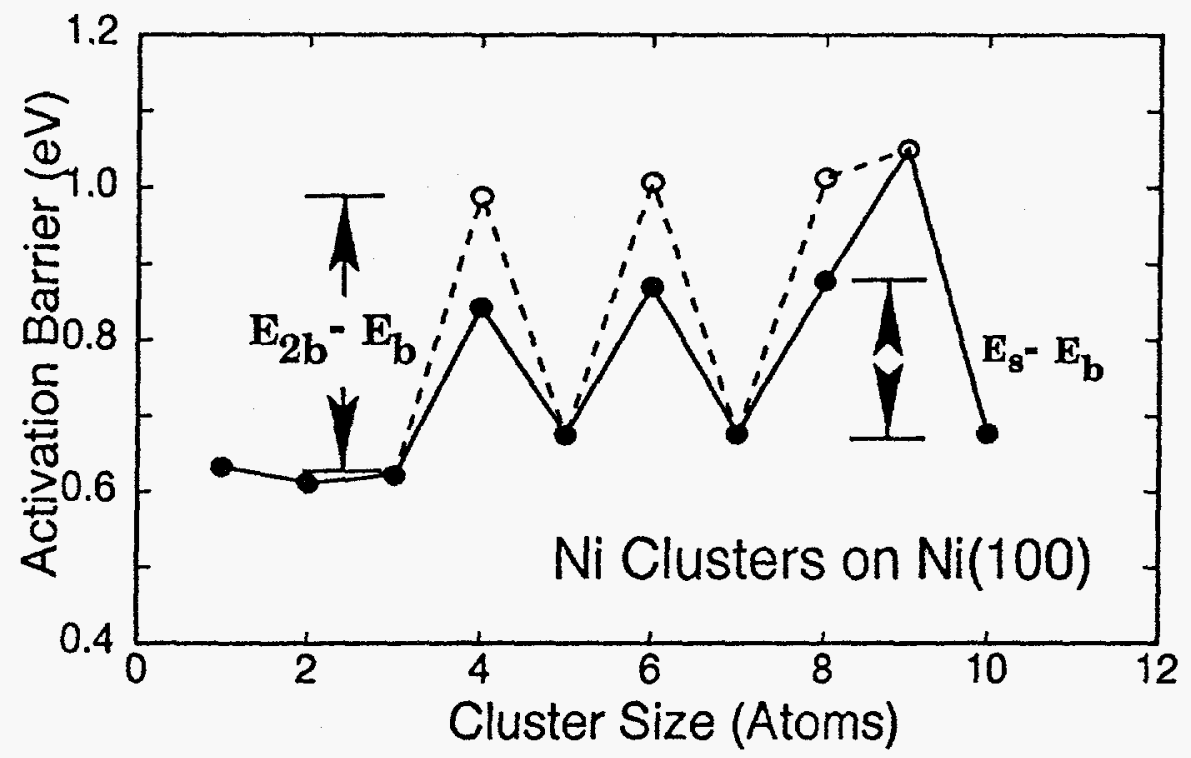

Fig. 3. A plot of the rate-limiting activation energy of surface diffusion for Ni islands on Ni(100). The solid curve corresponds to the case where dimer shearing is active, while the dashed curve is obtained with the restriction of sequential motion of individual atoms.

Table I. Activation energies (in $\mathrm{eV}$ ) of different atomic processes of diffusion or dissociation for adatom and islands of sizes 2 to 10 on the fcc (100) surface of three fcc metals.

\begin{tabular}{|c|c|c|c|}
\hline Cluster Geometry & $\mathrm{Cu}(100)$ & $\mathrm{Ag}(100)$ & $\mathrm{Ni}(100)$ \\
\hline & 0.503 & 0.478 & 0.632 \\
\hline & 0.494 & 0.480 & 0.611 \\
\hline & 0.501 & 0.491 & 0.621 \\
\hline$D$ & $0.688,0.815$ & $0.637,0.723$ & $0.842,0.989$ \\
\hline & 0.551 & 0.510 & 0.675 \\
\hline & $0.713,0.835$ & $0.658,0.737$ & $0.870,1.008$ \\
\hline & 0.552 & 0.512 & 0.677 \\
\hline & $0.717,0.841$ & $0.665,0.742$ & $0.875,1.013$ \\
\hline & $0.758,0.838$ & $0.676,0.740$ & $0.918,1.011$ \\
\hline & $0.857,0.975$ & $0.763,0.903$ & 1.051 .1 .215 \\
\hline & 0.554 & 0.516 & 0.678 \\
\hline
\end{tabular}


Consideration of dimer shearing also introduces interesting localized dynamics into some islands. For a compact octamer (a $3 \times 3$ island with one corner atom missing), there are four atomic processes shown in Fig. 4: (a) double-bond scission, (b) dimer sliding out, (c) dimer splitting, and (d) dimer sliding along the ledge. Notice that the last process has the lowest activation energy.

We expect a similar dimer sliding motion to be a favorable process along a true step edge as well, as confirmed by calculations. The results are shown in Table II for $\mathrm{Cu}(100)$, $\mathrm{Ag}(100)$ and $\mathrm{Ni}(100)$. For example, the activation energy for a $\mathrm{Ni}$ dimer sliding along the ledge on $\mathrm{Ni}(100)$ surface is $0.62 \mathrm{eV}$, which is lower than the energy barrier of $0.67 \mathrm{eV}$ to split the dimer at the ledge. It is also interesting to note that the barrier of $0.62 \mathrm{eV}$ for dimer sliding along a step edge is expectedly higher than the barrier of $0.34 \mathrm{eV}$ for single adatom diffusion along the step edge, but is still slightly lower than the barrier of $0.63 \mathrm{eV}$ for adatom diffusion on a terrace. This conclusion agrees qualitatively with recent observation of $\mathrm{Rh}$ adatom and $\mathrm{Rh}$ dimer at the step edge on $\mathrm{Rh}(100)$ surface. ${ }^{35}$

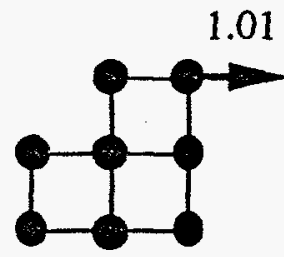

(a)

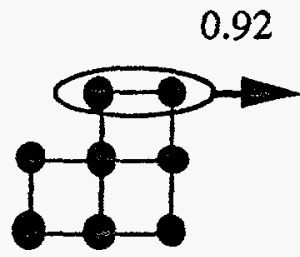

(b)

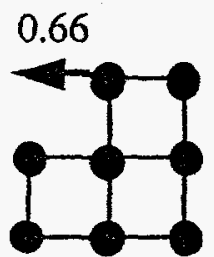

(c)

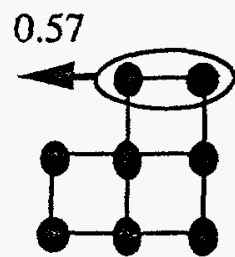

(d)

Fig. 4. Four atomic processes of an octamer on an fcc(100) surface: (a) double-bond scission; (b) dimer sliding out; (c) dimer splitting; and (d) dimer sliding along the ledge. The numbers are activation energies in units of $\mathrm{eV}$ as calculated for the case of $\mathrm{Ni}$.

Table II. Activation energies, in $\mathrm{eV}$, of adatom diffusion, dimer sliding, and dimer splitting along the ledge of a step on three fcc metal $(100)$ surfaces.

\begin{tabular}{c|c|c|c|}
\hline Geometry & $\mathrm{Cu}(100)$ & $\mathrm{Ag}(100)$ & $\mathrm{Ni}(100)$ \\
\hline
\end{tabular}

Next we explain how the shear motion of a dimer affects the process of nucleation and growth in (100) submonolayer epitaxy. In classical nucleation theory, ${ }^{36}$ the steady-state island density, $N$, which relates to the adatom diffusion constant $D$ and the deposition flux $F$, is described by

$$
N \sim\left(\frac{D}{F}\right)^{-p} \exp \left(\frac{E_{i}}{(i+2) k_{B} T}\right)
$$

where $p=i /(i+2)$ is the growth exponent, $i$ is the critical nucleus size defined by $i=s-1$ with $s$ the minimum size of the stable island, $E_{i}$ the cohesive energy of the critical-sized island, $T$ the surface temperature, and $\mathrm{k}_{\mathrm{B}}$ the Boltzmann constant. If a single dimer is stable, $i=1$, and $\mathrm{Eq}$. (1) reduces to $N \sim(D / F)^{-1 / 3}$. The regime $i=3$ has been suggested in several metal $(100)$ homoepitaxial growth systems at higher temperatures. ${ }^{37-40}$ Examining the role of dimer 
shearing, a new critical island size of $i=8 \mathrm{can}$ be defined. This new regime can be introduced using a hypothetical annealing experiment. First, we prepare a surface filled with a collection of two-dimensional islands of varying sizes, predominantly in their most compact configurations. Then we increase the substrate temperature to several specific values, each time for the same duration. When the surface temperature is sufficiently low, a dimer formed on the surface is stable, as are all larger islands. Therefore, the minimum size of the kinetically stable island is $s=2$. If the temperature is increased to the regime where single atom breaking is active but not dimer shearing, then only compact islands without an extra atom at the periphery are stable. In this case $s=4$. If the temperature is further increased to the regime where dimer shearing is active but not double-bond scission, then only islands with compact $3 \times 3$ structure are stable, because once a dimer shears out of a compact island of size 4,6 , or 8 , atom detachment by single bond scission can take place. In this case, $s=9$, and most of the surviving islands are at least 3-atoms wide. Finally, when the temperature is high enough to make double-bond scission an efficient process, then islands of all sizes can be dissociated. In this regime, either one large island will be formed on an ideally flat surface, or, more realistically, all the islands will join pre-existing steps. To this end, one recent experiment on $\mathrm{Cu}(100)$ submonolayer homoepitaxy has already provided confirmative support to the existence of $i=8.41$

\section{EFFECTS OF HYDROGEN ON ISLAND MOBILITY AND STABILITY}

In this section, we present a model study of a connected class of problems by investigating systematically the effects of impurities in the formation, migration, and dissociation of two-dimensional islands of various sizes. Our study is partly motivated by the desire to understand microscopically how surface impurities can dramatically alter the mobility and/or the stability of adatoms ${ }^{42,43}$ and islands. ${ }^{11,12}$ Such studies are also crucially required for understanding why in some circumstances surface impurities can function as surfactants in enhancing layer-by-layer growth, while in many other cases they induce rougher growth.

We choose $\mathrm{H}$ on $\mathrm{Ni}(100)$ as a specific example, and address various fundamentally important issues by classical-potential total-energy calculations with semiclassical hydrogen zero-point-energy (ZPE) corrections. Most of the calculations are done using a slab consisting of 77 dynamically active $\mathrm{Ni}$ atoms arranged in 3 layers and surrounded by a "basin" consisting of 2 layers of $\mathrm{Ni}$ atoms, rigidly held in equilibrium (100) positions, for a total of $322 \mathrm{Ni}$ atoms. Sample calculations have been made to make sure that the slab size is large enough to numerically converge the energy barriers for site-to-site mobility to better than $0.005 \mathrm{eV}$. The Ni island atoms are placed in the central area of the surface either with or without a $\mathrm{H}$ atom, all of which are also free to move. We use the embedded atom method (EAM) potential energy proposed by Daw and Baskes ${ }^{33}$ for this system as modified by Wonchoba, Hu, and Truhlar (EAM5 of Ref. 34). This potential is realistic in the sense that it has been parametrized to fit Ni-Ni equilibrium distances, bulk Ni elastic constants, $\mathrm{H}-\mathrm{Ni}$ equilibrium distances, surface binding energies, and hydrogen vibrational frequencies, with the result that it also gives reasonable barrier heights for adatom $\mathrm{H}$ diffusion and $\mathrm{Ni}$ diffusion. ${ }^{34}$

Energy barrier heights for various hopping processes are determined using an approach similar to that described in the previous section. What is special here is the inclusion of the effects of ZPE for the $\mathrm{H}$ atom, by calculating its classical vibrational frequencies at each step. If the hydrogen motion is unbound in any direction and an imaginary frequency results, the unbound coordinate is disregarded in the ZPE calculation. For several different $\mathrm{H}$ and $\mathrm{Ni}$ configurations we compared the classically estimated $\mathrm{H}$ atom ZPE to a fully quantum mechanical ZPE, computed by a fast Fourier transform technique, ${ }^{44}$ and found that the classical ZPE was consistently about $20 \%$ lower than the quantum ZPE. Therefore the errors in the semiclassical ZPE will tend to cancel out for the energy barriers reported here.

A $\mathrm{H}$ atom prefers the four-fold hollow site above the surface, with a site-to-site hopping barrier of $V_{H}=0.17 \mathrm{eV}$ for the EAM5 surface. A Ni adatom on a clean Ni(100) surface also prefers the four-fold hollow site, with a site-to-site hopping barrier of $V_{N i}=0.61$ $\mathrm{eV}$ for the EAM5 surface. Although there has been no systematic experimental study of self- 
diffusion on $\mathrm{Ni}(100)$, the value obtained here is near the value of $0.63 \mathrm{eV}$, the only available single-temperature estimate using a field ion microscope (FIM) ${ }^{42}$

Next we examine how a $\mathrm{H}$ adatom and a $\mathrm{Ni}$ adatom interact on the surface. There is a weak $(\sim 0.02 \mathrm{eV})$ attractive interaction between $\mathrm{H}$ and $\mathrm{Ni}$ when the two become nearest neighbors (i.e., occupying two nearest neighboring sites). This weak attraction is ineffective in slowing down the fast motion of $\mathrm{H}$, which can still easily jump away from the $\mathrm{Ni}$ with an activation barrier of $0.19 \mathrm{eV}$. But before the $\mathrm{H}$ atom hops away, it can significantly enhance the diffusion of the $\mathrm{Ni}$ atom via two different mechanisms. One is for the $\mathrm{H}$ to serve as a "stepping stone" to the $\mathrm{Ni}$ atom, which can easily jump on top of the $\mathrm{H}$, reside for a very short time, than jump over to the other side of the H (see Fig. 5). The activation barriers involved are $0.47 \mathrm{eV}$ and $0.067 \mathrm{eV}$, respectively, both of which are significantly lower than the diffusion barrier of $0.61 \mathrm{eV}$ for a lone Ni. In calculating the barriers, the variation of the $\mathrm{H}$-atom $\mathrm{ZPE}$ from $0.10 \mathrm{eV}$ to $0.15 \mathrm{eV}$ as the $\mathrm{Ni}$ hops on top of it has been included. In the other mechanism, the $\mathrm{Ni}$ and the $\mathrm{H}$ atoms move together as a pair by translating a distance equal to the surface lattice constant, with the $\mathrm{Ni}$ atom pushing from behind. The activation energy involved is also $0.47 \mathrm{eV}$.

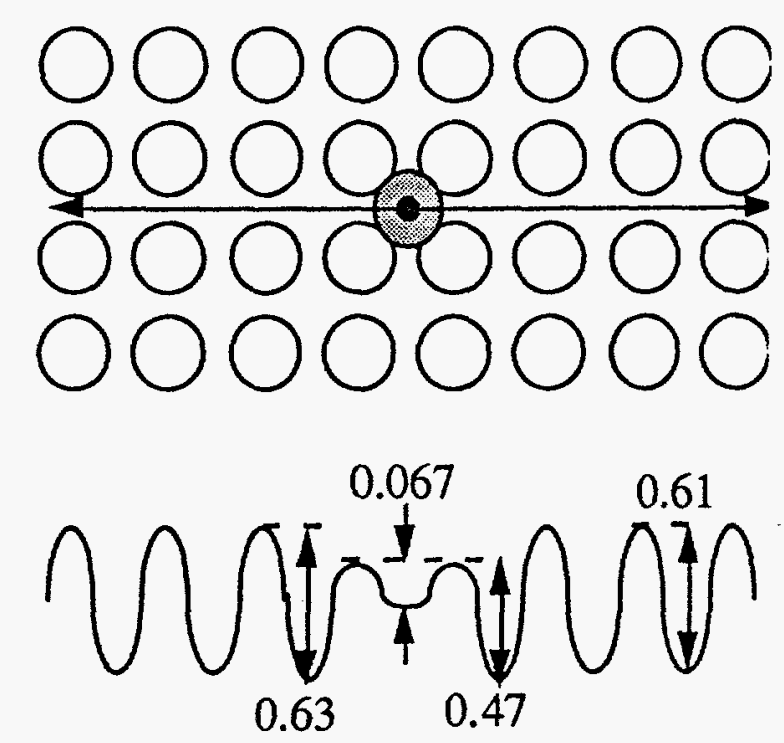

Fig. 5. A schematic representation of the H-enhanced Ni self-diffusion on $\mathrm{Ni}(100)$ via the "stepping-stone" mechanism. The large circles are $\mathrm{Ni}$ atoms, with the shaded one representing a Ni adatom sitting on top of a $\mathrm{H}$ atom (filled and smaller) located at a quasi-subsurface interstitial site. In the lower part, all activation energies are in $\mathrm{eV}$.

If a diffusing $\mathrm{H}$ adatom encounters a $\mathrm{Ni}$ island of any size, it can destabilize the island by reducing the activation barrier for a corner $\mathrm{Ni}$ atom nearest the $\mathrm{H}$ to leave the island and jump toward the $\mathrm{H}$. For example, the barrier for a corner atom to leave a compact $\mathrm{Ni}$ tetramer and hop toward a $\mathrm{H}$ atom (see Fig 6a) is $0.84 \mathrm{eV}$, which is lower than the corresponding barrier of $0.90 \mathrm{eV}$ for the same process without $\mathrm{H}$. The activation barrier for $\mathrm{H}$-assisted evaporation of a corner atom from a compact $3 \times 3 \mathrm{Ni}$ island also decreases, to $0.83 \mathrm{eV}$ from $0.90 \mathrm{eV}$ without $\mathrm{H}$, and for larger compact $m x n$ islands (with both $m$ and $n$ larger than 3) this trend remains unchanged.

It is illustrating to analyze the physical origin of the $\mathrm{H}$-enhanced mobility of a $\mathrm{Ni}$ adatom and the $\mathrm{H}$-reduced kinetic stability of a $\mathrm{Ni}$ island. When a $\mathrm{H}$ is residing in an equilibrium configuration as a nearest neighbor to a $\mathrm{Ni}$ atom (or a $\mathrm{Ni}$ island), the binding of the $\mathrm{H}$ atom to either the adatom or the island is weak. But when the nearest-neighbor $\mathrm{Ni}$ atom moves toward the $\mathrm{H}$, the $\mathrm{H}$ atom is lifted out of the well to meet the $\mathrm{Ni}$, resulting in a sizable reduction in transition-state energy. Qualitatively, the gain in activation energy results from the formation of a strengthened $\mathrm{Ni}-\mathrm{H}$ bond in the transition state, which partially compensates the energy loss as the $\mathrm{Ni}$ atom leaves the island by breaking two $\mathrm{Ni}-\mathrm{Ni}$ bonds.

The effect of $\mathrm{H}$ on island mobility is most significant at the comer of an island. As emphasized by Voter ${ }^{23}$ and more recently by Wen et al., ${ }^{48}$ the breakup of the compact $m x n$ core of a large island is the rate limiting process for long-range diffusion of the island under 
both PD and EC mechanisms. The easiest step for core breakup is to remove a corner atom. Once a corner is rounded, atoms can detach relatively easily from the kink sites, run along the island edges, and cause the center of mass of the island to change by the PD mechanism. Atoms released from the kink sites and running along the straight edges also find it easier to evaporate, causing the island to move by the EC mechanism.

(a)<smiles>[O]</smiles>

(c)

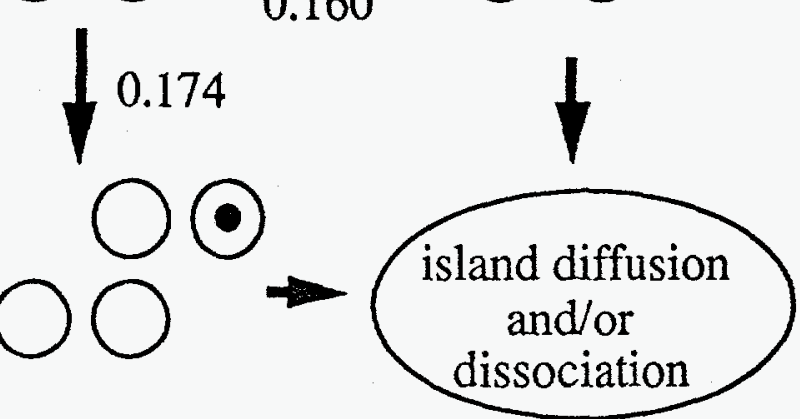

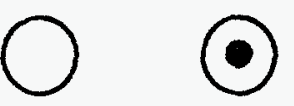

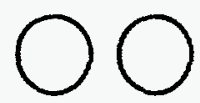

(b)

Fig. 6. H-assisted evaporation of a corner $\mathrm{Ni}$ atom from a compact $\mathrm{Ni}$ tetramer island. (a) Initial configuration, in which a hydrogen is attached to the island edge. (b) Final configuration, reached after one $\mathrm{Ni}$ atom leaves the island and pushes forward the $\mathrm{H}$ atom. (c) Final configuration, reached after two of the $\mathrm{Ni}$ atoms move collectively towards the $\mathrm{H}$ atom. Both configurations (b) and (c) can be followed by further diffusion and/or dissociation of the island.

The present finding, that a $\mathrm{H}$ adatom can significantly speed up the rate limiting process of core breakup by corner rounding, suggests that the presence of $\mathrm{H}$ will increase the mobility of $\mathrm{Ni}$ islands on $\mathrm{Ni}(100)$. This conclusion holds for $\mathrm{Ni}$ islands of all sizes, but different diffusion mechanisms may exist and compete for dominance for different-sized islands. The diffusion of large islands (containing tens of atoms or more) can be facilitated mainly by two nonexclusive mechanisms, PD and EC. ${ }^{8}$ Within these two mechanisms, the island diffusion coefficient can be expressed by $D_{P D} \sim N-3 / 2 \exp \left\{-V_{P D} / k T\right\}$ and $D_{E C} \sim N-1 / 2$ $\exp \left\{-V_{E C} / k T\right\}, 6,8$ where $N$ is the island size, $T$ the surface temperature, and $V_{P D}$ and $V_{E C}$ the effective activation barrier within the PD and EC mechanism, respectively. In the case where core breakup is the unique rate-limiting process for both the PD and the EC mechanisms, one has $V_{P D} \sim V_{E C}$, suggesting that the EC mechanism should always dominate when the island is sufficiently large. 8,9

We briefly discuss our results in connection with available experimental data, first on monomer diffusion. FIM studies have shown that $H$ hinders self-diffusion on $W(321),{ }^{43}$ but enhance self-diffusion on $\mathrm{Ni}(110){ }^{42} \mathrm{No}$ similar study has been done on $\mathrm{Ni}(100)$. The enhanced diffusion observed on the $\mathrm{Ni}(110)$ surface, and the similar effect predicted here on the $\mathrm{Ni}(100)$ surface, may however occur by different diffusion mechanisms. On the (100) surface, the diffusion mechanism is by normal hopping across a bridge site. In contrast, on the (110) surface Ni diffuses by concerted motion, ${ }^{42}$ although diffusion by the normal hopping mechanism may also take place.

Two experiments have reported an adsorbate-induced dramatic decrease in the stability of islands. One is by STM, ${ }^{11}$ the other by spot-profile low energy electron diffraction (SPALEED). ${ }^{12}$ The STM study found that the radius of a Au adatom or vacancy island formed in air on $\mathrm{Au}(111)$ decays in time according to the power law $r \propto\left(t_{0}-t\right)^{1 / 2}$, while islands formed in ultrahigh vacuum conditions stay essentially intact. Although the precise species of the adsorbate was not identified, the authors did suggest that the dominant mechanism is likely due to adsorbate-assisted atom detachment from the island edges, qualitatively consistent with the findings of the present study. A closer example is the SPA-LEED observation of the evolution of $\mathrm{Cu}$ islands on $\mathrm{Cu}(100)$ with and without $\mathrm{H}^{12}$ It has been shown that the selfdiffusion on $\mathrm{Cu}(100)$ is by normal hopping over a bridge site. ${ }^{45}$ Therefore, one would 
expect the conclusions reached here to be qualitatively applicable to the case of $\mathrm{H}$ on $\mathrm{Cu}(100)$. Indeed, using SPA-LEED, Walters et al. have observed that the average separation between the $\mathrm{Cu}$ islands grown on $\mathrm{Cu}(100)$ continues to increase after the $\mathrm{Cu}$ deposition in the presence of $\mathrm{H}$, while without $\mathrm{H}$ the islands once formed are stable.

As a final remark, we note that the primary effect of $\mathrm{H}$ impurities demonstrated here, namely, they collectively decrease the island density by increasing the average island size during growth, is just the opposite to what has been shown to be important in surfactantenhanced layer-by-layer growth. ${ }^{46-48}$ Therefore, we expect $\mathrm{H}$ to function as an antisurfactant in such systems. Preliminary results of multilayer growth of $\mathrm{Cu}$ on $\mathrm{Cu}(100)$, with and without $\mathrm{H}$, indeed demonstrate that $\mathrm{H}$ induces rougher growth. ${ }^{12}$

\section{SUMMARY}

Using bond-counting arguments and detailed EAM calculations, we have established a simple yet crucially important atomic process, shear motion of a dimer belonging to a compact island, for island diffusion and island dissociation in metal (100) submonolayer epitaxy. Consideration of this process necessitates the reevaluation of many important dynamical processes in such systems. It provides the easiest diffusion pathway for some compact islands, interprets better the oscillatory behavior of island mobility with island size, introduces a rich variety of localized island dynamics, and defines a new set of possible critical-sized islands.

We have further shown that the fast diffusion of $\mathrm{H}$ on a flat $\mathrm{Ni}(100)$ surface is preserved even when the surface is decorated with various intrinsic defects such as $\mathrm{Ni}$ adatoms, islands, and steps created during epitaxial growth. On the other hand, the presence of $\mathrm{H}$ impurities on such a surface will significantly enhance the mobilities of both Ni adatoms and $\mathrm{Ni}$ islands, resulting in a decrease in island density and a corresponding increase in average island size. The net effect of the $\mathrm{H}$ is to function as an anti-surfactant in $\mathrm{Ni}(100)$ homoepitaxy. Similar phenomena are expected to exist in other metal (100) epitaxial growth systems in which the diffusion of the host adatom is by normal hopping over bridges, as demonstrated experimentally for the case of $\mathrm{Cu}(100)$ homoepitaxy.

ACKNOWLEDGEMENTS: We acknowledge the contributions of Anna Swan, John Wendelken, David John, Curtis Walters, David Zehner, and Ward Plummer to the work reviewed here. This work was mainly supported by Oak Ridge National Laboratory, managed by Lockheed Martin Energy Research Corp. for the U.S. Department of Energy under contract number DE-AC05-960R22464, in part by an appointment to the Oak Ridge National Laboratory Postdoctoral Research Associates-Program administrated by the Oak Ridge Institute for Science and Education (Z.P.S.), and by a grant from Lehigh University (K.H.).

\section{REFERENCES}

1. K. Stolt, W. R. Graham, and G. Ehrlich, J. Chem. Phys. 65, 3206 (1976).

2. D. W. Bassett, J. Phys. C. (Solid State Phys.) 9, 2491 (1976).

3. T. T. Tsong and R. Casanova, Phys. Rev. B 22, 4632 (1980).

4. S. C. Wang and G. Ehrlich, Surf Sci. 239, 301 (1990).

5. G. L. Kellogg, Phys. Rev. Lett. 73, 1833 (1994). For more references on FIM studies of island diffusion, see the recent review by Kellogg in: Surf. Sci. Reports 21 (1/2), (1994).

6. D. J. Trevor and C. E. D. Chidsey, J. Vac. Sci. Technol. B 9, 964 (1991).

7. J. de la Figuera, J. E. Pristo, O. Ocal, and R. Miranda, Solid State Commun. 89, 815 (1994).

8. J.-M. Wen, S.-L. Chang, J. W. Burnett, J. W. Evans, P. A. Thiel, Phys. Rev. Lett. 73, 2591 (1994).

9. K. Morgenstern, G. Rosenfeld, B. Poelsema, and G. Comsa, Phys. Rev. Lett. 74, 2058 (1995).

10. W. W. Pai, A. K. Swan, J. F. Wendelken, Z. Y. Zhang, to be published.

11. D. R. Peale and B. H. Cooper, J. Vac. Sci. Technol. A 10, 2210 (1991). 
12. C. F. Walters, Z. Y. Zhang, D. M. Zehner, and E. W. Plummer, presented at the 55th Conference on Physical Electronics, Flagstaf, Arizona, June 12-14, 1995; and to be published.

13. W. F. Egelhoff, Jr. and D. A. Steigerwald, J. Vac. Sci. Technol. A 7, 2167 (1989).

14. M. Copel, M. C. Reuter, E. Kaxiras, and R. M. Tromp, Phys. Rev. Lett. 63, 632 (1989).

15. H. A. van der Vegt, H. M. van Pinxteren, M. Lohmeier, E. Vlieg, and J. M. C. Thornton, Phys. Rev. Lett. 68, 3335 (1992).

16. M. Horn-von Hoegen, B.H. Muller, A. Al-Falou, and M. Henzler, Phys. Rev. Lett. 71, 3170 (1994). M. Horn-von Hoegen and A. Golla, Phys. Rev. Lett. 76, 2953 (1994).

17. S. Esch, M. Hohage, Th. Michely, and G. Comsa, Phys. Rev. Lett. 72, 518 (1994).

18. J. Vrijmoeth, H. A. van der Vegt, J. A. Meyer, E. Vlieg, and R. J. Behm, Phys. Rev. Lett. 72, 3843 (1994).

19. M. Copel and R. M. Tromp, Rev. Lett. 72, 1236 (1994); 76, 2603 (1996).

20. J. E. Vasek, Z. Y. Zhang, C. T. Salling, and M. G. Lagally, Phys. Rev. B 51, 17207 (1995).

21. P. J. Feibelman, Phys. Rev. Lett. 58, 2766 (1987).

22. T. Yamasaki, T. Uda, and K. Terakura, Phys. Rev. Lett. 76, 2949 (1996).

23. A. F. Voter, Phys. Rev, B 34, 6819 (1986).

24. C. L. Liu, J. M. Cohen, J. B. Adams, and A. F. Voter, Surf. Sci. 253, 334 (1991).

C. L. Liu and J. B. Adams, Surf. Sci. 268, 73 (1992); Surf. Sci. 316, 294 (1994).

C. L. Liu, Int. J. Mod. Phys. B 9, 1 (1995).

25. S. D. Liu, Z. Y. Zhang, J. K. Norskov, and H. Metiu, Surf Sci. 321, 161 (1994).

26. Z. P. Shi, Z. Y. Zhang, A. K. Swan, and J. F. Wendelken, Phys. Rev, Lett. 76, 4927 (1996).

27. D. S. Sholl and R. T. Skodje, Phys. Rev. Lett. 75, 3158 (1995).

28. J. C. Hamilton, M. S. Daw, and S. M. Foiles, Phys. Rev. Lett. 74, 2760 (1995).

29. C. DeW. Van Siclen, Phys. Rev. Lett. 75, 1574 (1995).

30. S. V. Khare, N. C. Bartelt, and T. L. Einstein, Phys. Rev. Lett. 75, 2148 (1995).

31. K. Haug, Z. Y. Zhang, D. T. John, C. F. Walters, D. M. Zehner, and E. W. Plummer, (submitted to Phys. Rev. Lett.) (1996).

32. R. Stumpf, Phys. Rev. B 53, R4253 (1996); and to be published.

33. M. S. Daw and M. I. Baskes, Phys. Rev. B 29, 6443 (1984).

34. S. E. Wonchoba, W. H. Hu, and D.G. Truhlar, Phys. Rev. B 51, 9985 (1995).

35. G. L. Kellogg, Surf. Sci. 359, 237 (1996).

36. J. A. Venables, Philos. Mag. 27, 697 (1973); J. A. Venables, G. D. T. Spiller and M. Hanbucken, Rep. Prog. Phys. 16, 399 (1984).

37. J. K. Zuo, J. F. Wendelken, A. Durr and C. L. Liu, Phys. Rev. Lett. 72, 3064 (1994).

38. J. Stroscio and D. Pierce, Phys. Rev. B 49, 8522 (1994).

39. S. Günther, E. Kopatzki, M. C. Bartelt, J. W. Evans, and R. J. Behm, Phys. Rev. Lett. 73, 553 (1994).

40. M.C. Bartelt, L. S. Perkins, and J.W Evans, Surf. Sci. Lett. 344, L1193 (1995).

41. A. K. Swan, Z. P. Shi, Z. Y. Zhang, and J. F. Wendelken, (submitted) (1996).

42. R. T. Tung and W. R. Graham, Surf. Sci. 97, 73 (1980).

43. R. Casanova and T. T. Tsong, Surf. Sci. 94, L179 (1980).

44. K. Haug and H. Metiu, J. Chem. Phys. 94, 3251 (1991).

45. C. Lee, G. T. Barkema, M. Breeman, A. Pasquarello, and R. Car, Surf. Sci. Lett. 306, L575 (1994).

46. S. Oppo, V. Fiorentini, and M. Scheffler, Phys. Rev. Lett. 71, 2437 (1993).

47. Z. Y. Zhang and M. G. Lagally, Phys. Rev. Lett. 72, 693 (1994).

48. S. Liu, L. Bonig, J. Detch, and H. Metiu, Phys. Rev. Lett. 74, 4495 (1995). 